\title{
Lodo gerado na estação de tratamento de água Tamanduá, Foz do Iguaçu, PR, como aditivo em argilas para cerâmica vermelha. Parte I: Caracterização do lodo e de argilas do terceiro planalto paranaense
}

\author{
(Generated sludge at water treatment station Tamanduá, \\ Foz do Iguaçu, PR, as additive in red clay for ceramics. \\ Part I: Characterization of sludge and clay Paraná third plateau)
}

\author{
R. Tartari ${ }^{1,4}$, N. Díaz-Mora ${ }^{3}$, A. N. Módenes ${ }^{1}$, S. A. Pianaro ${ }^{2}$ \\ ${ }^{1}$ Departamento de Engenharia Química, Universidade Estadual do Oeste do Paraná - UNIOESTE, Rua da \\ Faculdade 645, Campus de Toledo, PR \\ ${ }^{2}$ Laboratório Interdisciplinar de Materiais Cerâmicos, Departamento de Engenharia de Materiais, Universidade \\ Estadual de Ponta Grossa - UEPG, Rua Carlos Cavalcanti 4748, Campus de Uvaranas, Ponta Grossa, PR \\ ${ }^{3}$ Laboratório de Materiais - LAMAT, Centro de Engenharias e Ciências Exatas, Universidade Estadual do Oeste \\ do Paraná - UNIOESTE, Rua Tarquínio Joslin Santos 1300, Campus de Foz do Iguaçu, PR \\ ${ }^{4}$ Instituto de Educação, Agricultura e Ambiente, Engenharia Ambiental, Universidade Federal do Amazonas - \\ UFAM, Rua 29 de Agosto 786, Campus Vale do Rio Madeira, Humaitá, AM \\ engrt84@yahoo.com.br
}

\begin{abstract}
Resumo
Com a finalidade de pesquisar a incorporação do lodo gerado na ETA Tamanduá em massas para cerâmica vermelha, como método de destinação final, o presente artigo tem como objetivo caracterizar o lodo da ETA Tamanduá e quatro argilas da região Oeste do Paraná utilizadas na produção de cerâmica vermelha estrutural e de artesanato, visando avaliar o potencial tecnológico deste resíduo. Nessa primeira etapa experimental de caracterização, determinaram-se a umidade, composição química por fluorescência de raios X, compostos cristalinos por difração de raios X, limites de consistência de solos (Atterberg), distribuição granulométrica e a estrutura das partículas por microscopia eletrônica de varredura (MEV). Destaca-se que as argilas possuem propriedades favoráveis para produção de cerâmica vermelha. Já o lodo, possui características elementares de matéria-prima de baixa plasticidade, composto majoritariamente por $\mathrm{SiO}_{2}$, $\mathrm{Al}_{2} \mathrm{O}_{3}$ e $\mathrm{Fe}_{2} \mathrm{O}_{3}$ com perda ao fogo elevada $(20,4 \%$ ), baixo índice de plasticidade igual a $(7,4)$, fração de argila correspondentes a 5,3\%. As características observadas sugerem a possibilidade de, na segunda etapa, incorporar o lodo com umidade natural (75\%) como aditivo na massa cerâmica, visando reduzir a retração, bem como minimizar a adição de água às massas para produção de cerâmica vermelha.

Palavras-chave: lodo de estação de tratamento de água, caracterização de argilas, resíduos.
\end{abstract}

\begin{abstract}
With a view to investigate the incorporation of sludge generated in ETA Tamanduá masses for red ceramics, such as disposal method, this paper aims to characterize the sludge ETA Tamanduá and four clays of western Paraná used to produce structural red ceramic and artistic ceramic, to evaluate the technological potential of this residue. In this first experimental phase of characterization, we determined the moisture, chemical composition by X-ray fluorescence, crystalline compounds by X-ray diffraction, Atterberg limits of soils (Atterberg), size distribution and structure of particles by electron microscopy (SEM). It is noteworthy that the clays have properties favorable for the production of red ceramics. The sludge has basic features of the raw material of low plasticity, composed mainly of $\mathrm{SiO}_{2}, \mathrm{Al}_{2} \mathrm{O}_{3}$ and $\mathrm{Fe}_{2} \mathrm{O}_{3}$, with high loss on ignition (20.4\%), low plasticity index equal to (7.4), fraction clay corresponding to 5.3\%. These characteristics suggested the possibility that, in the second stage, incorporate the sludge with natural moisture (75\%) as an additive in ceramic body, to reduce the shrinkage and to minimize the addition of water to the masses for the production of red ceramics.
\end{abstract}

Keywords: sludge from water treatment plants, characterization of clays, west .

\section{INTRODUÇÃO}

O processo convencional de tratamento e purificação da água tem sido adotado pelo setor de saneamento com a principal finalidade de retirar impurezas presentes na água originárias da formação geológica do manancial e de atividades poluidoras ativas inseridas na área de influência. Neste sistema, obtém-se água potável como produto final, e o subproduto lodo de Estação de Tratamento de Água (ETA), formado por sólidos e precipitados químicos, constituindo uma massa de partículas orgânicas e inorgânicas, densa e viscosa [1]. As características quali-quantitativas do 
lodo gerado podem variar conforme o gerenciamento do processo de tratamento, métodos de operação do sistema, periodicidade de limpeza dos decantadores e filtros e da dosagem de produtos químicos que é função da variação temporal das características físico-químicas da água de captação, a qual está associada à formação geológica onde o manancial está inserido, ao uso e a ocupação do solo da área da bacia hidrográfica e ao ciclo hidrológico característico regional. Os lodos de ETA, contêm concentrações elevadas de elementos químicos ( $\mathrm{Al}, \mathrm{Fe}, \mathrm{Si}, \mathrm{Ti}, \mathrm{Mg}$, entre outros) que, quando dispostos de forma inadequada, podem gerar impactos de ordem ambiental e social $[1,2]$.

A regulamentação sobre a preservação e qualidade ambiental, tem interferido positivamente na atuação das empresas de saneamento, as quais têm criado estímulos para o adequado gerenciamento e disposição final desse resíduo, consolidando a co-responsabilidade e o comprometimento no manejo dos recursos naturais. A empresa de saneamento do Paraná - Sanepar, a exemplo de outras empresas no país, com o intuito de minimizar impactos ambientais causados pela atividade, está buscando desenvolver métodos de disposição final deste resíduo, com base nos requisitos técnicos e com viabilidade econômica, o que caracteriza ação imediata para aperfeiçoamento e melhoria contínua do sistema de gestão ambiental. Dentre as medidas adotadas e em estudo pela empresa, se destaca a gestão estratégia e operacional de lodo de ETA, avaliação do processo de coagulação e floculação, avaliação do limite de aplicação de lodo em solo, mistura de lodo em solo-cimento e incorporação de lodo em argilas para produção de cerâmica vermelha. Pesquisas desenvolvidas na área estão orientadas ao reuso de lodos resultantes do processo de tratamento de água, desidratação química, quantificação, impactos do lançamento in natura em cursos d'água, disposição em matriz de concreto, incorporação em cerâmica vermelha e análise dos gases poluentes liberados durante o processo de sinterização [3-11]. Devido ao fato das massas argilosas utilizadas em grande parte da indústria cerâmica serem por natureza heterogêneas e apresentarem variação física, química e mineralógica, semelhanças com as características do lodo, têm sido apontadas como uma possível rota de utilização desses resíduos [3] mediante sua incorporação em cerâmica vermelha $[8,10]$.

O setor cerâmico na região Oeste do Paraná está fortalecido no mercado, comprometido com pesquisas tecnológicas para produção de novos produtos ecológicos, contendo resíduos em suas matrizes de cerâmica vermelha. Por tanto, este trabalho tem por objetivo principal caracterizar o lodo gerado na ETA Tamanduá de Foz do Iguaçu/PR e de quatro (4) argilas do terceiro planalto Paranaense, visando avaliar seu potencial tecnológico e identificar as condições específicas de utilização como aditivo em cerâmica vermelha que será investigado na Etapa 2 deste trabalho, o que permitirá atender às normas/ legislações, contribuir com o controle da qualidade do produto, com a viabilidade financeira e o impacto ambiental agregado.

\section{Processo de tratamento de água e geração de lodo}

A Companhia de Saneamento do Paraná, Unidade Regional de Foz do Iguaçu, PR (URFI) opera a ETA instalada no rio Tamanduá, Rodovia 469, Foz do Iguaçu, PR. Para atender a aproximadamente 140 mil habitantes, $45 \%$ da população do município, faz-se a captação em média de $250 \mathrm{~L} / \mathrm{s}$ de água para tratamento convencional, conforme ilustrado no fluxograma do processo de tratamento de água e geração de lodos (Fig. 1). No processo, é adicionado à água o coagulante policloreto de alumínio (PAC), inorgânico, catiônico, pré-polimerizado, que garante a desestabilização das partículas em suspensão para união catiônica, garantindo a formação de flocos com maior peso e a precipitação se torna mais rápida e eficiente devido à velocidade de formação dos flocos serem superiores quando comparados aos coagulantes tradicionais não pré-polimerizados [12]. A ETA Tamanduá opera com quatro floculadores que induz a aglomeração dos sólidos presentes na água, formando flocos que seguem seu fluxo em direção aos decantadores de alta taxa, e por ação da gravidade, precipitam e acondicionam-se no fundo. $\mathrm{Na}$ etapa de filtração, as impurezas ainda presentes são retidas em 12 filtros convencionais existentes, formando uma manta de lodo e a água clarificada direcionada às fases de desinfecção e correção do $\mathrm{pH}$, para posteriormente serem aduzidas ao reservatório de distribuição. As etapas de decantação e filtração são responsáveis pela geração do lodo bruto, que é encaminhado pela descarga a uma câmara de equalização e posteriormente, bombeada ao adensador que por ação cinética de movimento, separa a fase líquida da fase sólida. Os líquidos e sobrenadantes presentes na superfície do adensador são retornados para a fase inicial do processo. Do adensador, o lodo formado é descarregado pelo fundo e encaminhado ao processo de centrifugação onde recebe uma determinada concentração de polímero comercial. No final do processo, o material sólido centrifugado é depositado em um contêiner, onde permanece em tempo de espera para destinação no aterro sanitário municipal de Foz do Iguaçu, PR, de acordo com procedimento atual da empresa.

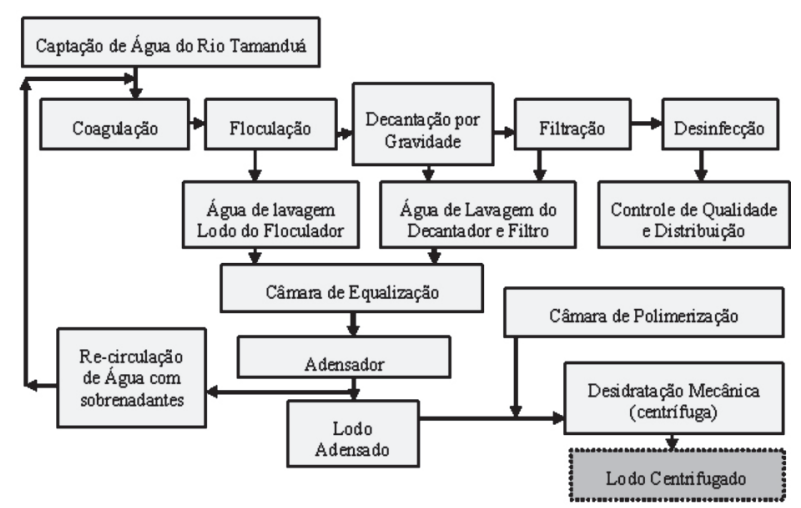

Figura 1: Processo convencional de tratamento de água da ETA Tamanduá.

[Figure 1: Conventional process water treatment WTS Tamanduá.] 


\section{MATERIAIS E MÉTODOS}

\section{Coleta e amostragem das matérias-primas}

Neste trabalho, foram utilizadas além do lodo gerado na ETA Tamanduá, quatro argilas distintas, sendo duas daquelas utilizadas na preparação de massa para cerâmica artística na Cooperativa de Artesões de Foz do Iguaçu, PR (COOAFOZ) denominadas argilas AP e AA e duas argilas denominadas OP e OA, empregadas na preparação da massa industrial para produção de tijolos pela Empresa Cerâmica Santa Rita. Tais argilas são exploradas na região Oeste do Paraná de formação geológica do terceiro planalto paranaense da era mesozóica [22], há aproximadamente 40 anos, utilizadas na fabricação de materiais cerâmicos [13]. Foram coletados 100 $\mathrm{kg}$ do lodo acumulado em todos os eventos de centrifugação no período de quatro meses e armazenados em um contêiner de polietileno protegido das intempéries. A coleta realizada em cada lote de argila armazenada nos pátios em tempo de maturação procedera-se segundo recomendações técnicas [20], totalizando $50 \mathrm{~kg}$ de cada.

\section{Caracterização das matérias-primas}

Todas as materiais-primas foram submetidas ao processo de secagem em estufa até peso constante $\left(110 \pm 5^{\circ} \mathrm{C}\right)$, destorroamento, homogeneização e peneiramento, conforme o procedimento de preparação de amostras de solos, [14]. Para caracterização física, química e mineralógica, determinaram-se: o teor de umidade (\%) [15], perda ao fogo (\%) [16], índices de consistência de solos de Atterberg - limites de liquidez [17] e plasticidade [18]. Utilizouse o granulômetro a laser Cilas 920 para caracterização granulométrica. A composição química foi determinada pela técnica de fluorescência de raios X por Energia Dispersiva (ED-FRX), método do pó, em equipamento Shimadzu 700. A caracterização microestrutural das partículas de lodo foi realizada por microscopia eletrônica de varredura (MEV) e a microanálise por Energia Dispersiva por fluorescência de raios X (EDS) em microscópio Shimadzu SSV-550. A caracterização mineralógica foi realizada pela técnica de difração de raios X [19] em difratômetro Shimadzu XRD 700 , ânodo rotatório de cobre. A varredura foi de 5 a $60^{\circ}(2 \theta)$, com passo $0,02^{\circ}$ e velocidade de varredura $2 \%$ min. As fases cristalinas foram identificadas com base nas informações da biblioteca JCPDS-700.

\section{RESULTADOS E DISCUSSÃO}

O lodo da ETA Tamanduá, após o processo de adensamento e centrifugação apresentou $74 \pm 3 \%$ de umidade, sendo o restante, sólidos totais, que representa concentração satisfatória para desidratação mecânica por centrifugação, conforme especificações técnicas que indicam a faixa ideal de 16 a 35\% [1]. Os teores de umidade das argilas nas condições ambientais locais de acondicionamento e armazenamento foram: $\mathrm{AP}(15,8 \pm 0,8 \%), \mathrm{AA}(21,3 \pm 0,7 \%)$, OA $(23,5 \% \pm 0,4 \%)$ e OP $(32,2 \% \pm 0,3 \%)$. A composição química das matérias-primas é apresentada na Tabela I, e proporciona suporte para análise mineralógica obtida por difração de raios $\mathrm{X}$ mostrada na Fig. 2. Observa-se que as argilas são compostas majoritariamente por $\mathrm{SiO}_{2}, \mathrm{Al}_{2} \mathrm{O}_{3}$, $\mathrm{Fe}_{2} \mathrm{O}_{3}$ e $\mathrm{TiO}_{2}$, e em menores concentrações encontraram-se os óxidos alcalino $\mathrm{K}_{2} \mathrm{O}$ e alcalino terroso $\mathrm{CaO}$, que atuam como agentes fundentes ativos no processo de sinterização de materiais cerâmicos, normalmente em temperaturas superiores a $1000{ }^{\circ} \mathrm{C}$. A composição química do lodo é formada por $74 \%$ dos óxidos de $\mathrm{SiO}_{2}, \mathrm{Al}_{2} \mathrm{O}_{3}$ e $\mathrm{Fe}_{2} \mathrm{O}_{3}$. A perda ao fogo de $20,4 \%$ se deve à presença de águas intersticiais de coordenação zeolítica, hidroxilas dos argilominerais e dos hidróxidos existentes, como também, pode originar-se parcialmente dos componentes de matéria orgânica volátil [20]. Já as argilas, apresentaram valores de perda ao fogo, variando entre 6,2 e $12,8 \%$, porém, em menores percentuais quando comparados com a do lodo bruto. Destaca-se que a composição do coagulante utilizado no processo de tratamento de água agregou-se ao lodo, refletindo diretamente na composição majoritária de alumínio.

Foram identificadas nos difratogramas apresentados na Fig. 2, as fases cristalinas de três principais minerais: a caulinita, mineral de silicato de alumínio hidratado $\left(\mathrm{Al}_{4} \mathrm{SiO}_{4} \mathrm{O}_{10}(\mathrm{OH})_{8}\right)$, o quartzo, mineral de óxido de silício $\left(\mathrm{SiO}_{2}\right)$ e a hematita, mineral de óxido de ferro $\left(\mathrm{Fe}_{2} \mathrm{O}_{3}\right)$. Destaca-se que as argilas são de origem geológica do terceiro planalto paranaense da era mesozóica, formação por derrames de lavras vulcânicas de rochas do subsolo que deram origem a argilominerais por ação de intempéries a partir dos minerais alumino-silicátos pré-existentes, bem

Tabela I - Composição química do lodo de estação de tratamento de água e das argilas da região oeste do Paraná (\% peso). [Table I - Chemical composition of the water treatment plant sludge and clay from west the Parana (wt.\%)

\begin{tabular}{|c|c|c|c|c|c|c|c|c|c|c|}
\hline \multicolumn{2}{|c|}{ Matérias - primas } & \multirow{2}{*}{$\frac{\mathrm{Al}_{2} \mathrm{O}_{3}}{7,2}$} & \multirow{2}{*}{$\frac{\mathrm{Fe}_{2} \mathrm{O}_{3}}{7,3}$} & \multirow{2}{*}{$\frac{\mathrm{SiO}_{2}}{63,6}$} & \multirow{2}{*}{$\frac{\mathrm{TiO}_{2}}{12,1}$} & \multirow{2}{*}{$\frac{\mathrm{CaO}}{0,5}$} & \multirow{2}{*}{$\frac{\mathrm{SO}_{3}}{2,2}$} & \multirow{2}{*}{$\frac{\mathrm{ZrO}_{2}}{0,2}$} & \multirow{2}{*}{$\frac{\mathrm{K}_{2} \mathrm{O}}{0,7}$} & \multirow{2}{*}{$\begin{array}{c}\text { Perda ao } \\
\text { Fogo }(\%) \\
6,2\end{array}$} \\
\hline \multirow{4}{*}{ Argilas } & AA & & & & & & & & & \\
\hline & AP & 19 & 10,7 & 42,8 & 14,1 & 1,0 & 2,1 & - & 0,8 & 9,5 \\
\hline & $\mathrm{OA}$ & 10,7 & 29,6 & 36,1 & 13,9 & 1,0 & 1,5 & 0,4 & 0,4 & 6,8 \\
\hline & OP & 26,5 & 9,3 & 40,2 & 8,4 & 0,5 & 2,3 & - & - & 12,8 \\
\hline \multicolumn{2}{|c|}{ Lodos - ETA Tamanduá } & 31,6 & 18,6 & 24,1 & 2,2 & - & 2,8 & - & 0,3 & 20,4 \\
\hline
\end{tabular}

Nota: foram detectados elementos traços dos seguintes óxidos: $\mathrm{CuO}, \mathrm{CrO}_{2}, \mathrm{~V}_{2} \mathrm{O}_{5}, \mathrm{MnO}$. 


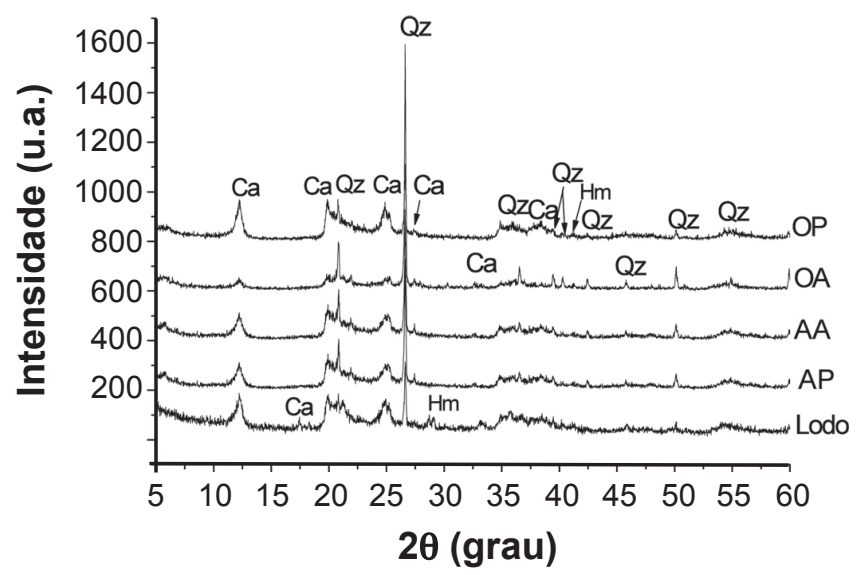

Figura 2: Difratograma de raios $\mathrm{X}$ das cinco materiais-primas: lodo, argilas AP, AA, OP e OA $(\mathrm{Ca}=$ Caulinta $; \mathrm{Qz}=$ Quartzo; $\mathrm{Hm}$ $=$ Hematita . .

[Figure 2: X-ray diffraction patterns of the five raw materials: sludge, clays $A P ; A A ; O P$ and $O A(C a=$ Caulinite; $Q z=$ Quartz; $\mathrm{Hm}=$ Hematite).]

como, por evoluções "in situ", por formação sedimentar de determinados perfis de solos, e por eventos de inundações, cuja ação dos grandes rios, Paraná e Iguaçu transportaram minerais que se depositaram nas áreas de planície de inundação nos eventos hidrológicos históricos, formando bolsões de solos argilosos de classificação Gley, onde nestes locais atualmente faz-se a lavra do recurso mineral para produção de cerâmica vermelha na região [13].

A análise de Atterberg mostrou que as argilas AP e OP possuem características inorgânicas de alta plasticidade (IP $=54$ e 47, respectivamente), com presença de argilominerais hidrofílicos que contribuem para forte ligação química, bem como, pela influência da fração dos grãos na faixa de 19,6 e $21,4 \%<2 \mu \mathrm{m}$ e baixa porcentagem de grãos do tamanho da areia, 16,2 e 21,5\%, conforme apresentados nas Figs. 3 e 4. Admite-se por essas características que a formulação da massa será constituída por partículas de distintos tamanhos, o que reduzirá o grau de compactação e os canais internos na peça cerâmica, favorecendo assim a eliminação das águas associadas às partículas em função do tempo no processo de secagem, de forma a garantir baixo gradiente diferencial

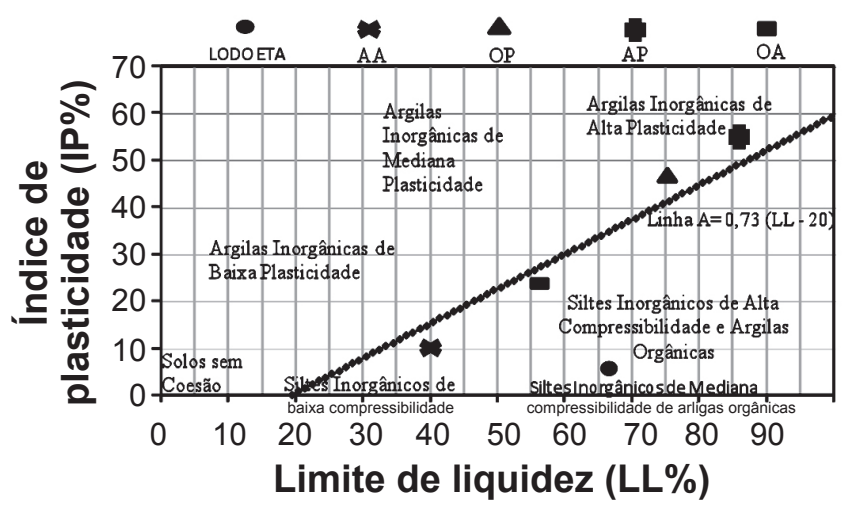

Figura 3: Limites de plasticidade e liquidez (Atterberg). [Figure 3: Limits of plasticity and liquidity (Atterberg).] de deformação linear. As características de alta plasticidade destas argilas podem ter relações diretas que indicam que as partículas sejam de morfologia lamelar, pois essa morfologia possui clivagem definida e orienta a molécula de água para ação lubrificante entre as partículas, facilitando o deslocamento das placas umas sobre as outras, logo, quando uma tensão tangencial for aplicada haverá maior plasticidade do que as que não as tem clivagem definida, favorecendo para consistência da estrutura sólida sem deformações e trincas após processamento [21].

A argila OA possui baixo índice de plasticidade (IP = 25), classificando-se como sendo uma argila constituída por siltes inorgânicos de alta compressibilidade, com $17 \%$ de grãos $<2 \mu \mathrm{m}$, o que favorece a formulação da massa e o empacotamento das partículas na conformação da peça cerâmica. A argila AA se destacou pelo elevado percentual de $\mathrm{SiO}_{2}(63,6 \%), 15 \%$ das partículas com granulometria $<2 \mu \mathrm{m}$, classificada como siltes inorgânicos de baixa compressibilidade. Nos estudos de consistência de solos de Atterberg, verificou-se que as melhores propriedades de plasticidade encontraram-se nas argilas AP seguida da OP, pois, ambas possuem indícios ideais de propriedades que potencializam sua utilização em maiores quantidades nas massas para cerâmica vermelha conforme propriedades de consistência apresentado na Fig. 3. Ressalta-se que a adição de lodo em argilas com características "gordas", de alta plasticidade, em porcentagens adequadas poderá aperfeiçoar a organização de partículas na peça cerâmica de forma a reduzir tempo e otimizar a eficácia no processo de secagem, uma vez que o empacotamento dos grãos no corpo cerâmico reduzirá a forte interação entre as partículas que adsorvem água para os interstícios.

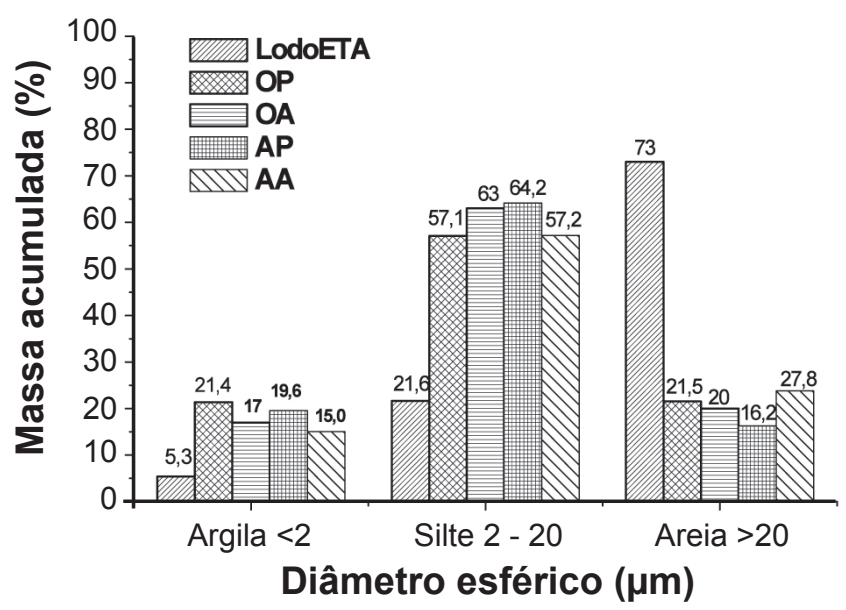

Figura 4: Distribuição granulométrica das matérias-primas. [Figure 4: Grain size distribution of the raw materials.]

Nas micrografias apresentadas nas Figs. 5, 6 e 7 observamse as morfologias típicas dos grãos do lodo de ETA [3,5], com estrutura formada por conglomerados de partículas de distintos tamanhos com aparência porosa, unidas por forças fracas, que originam partículas com distintos tamanhos, 

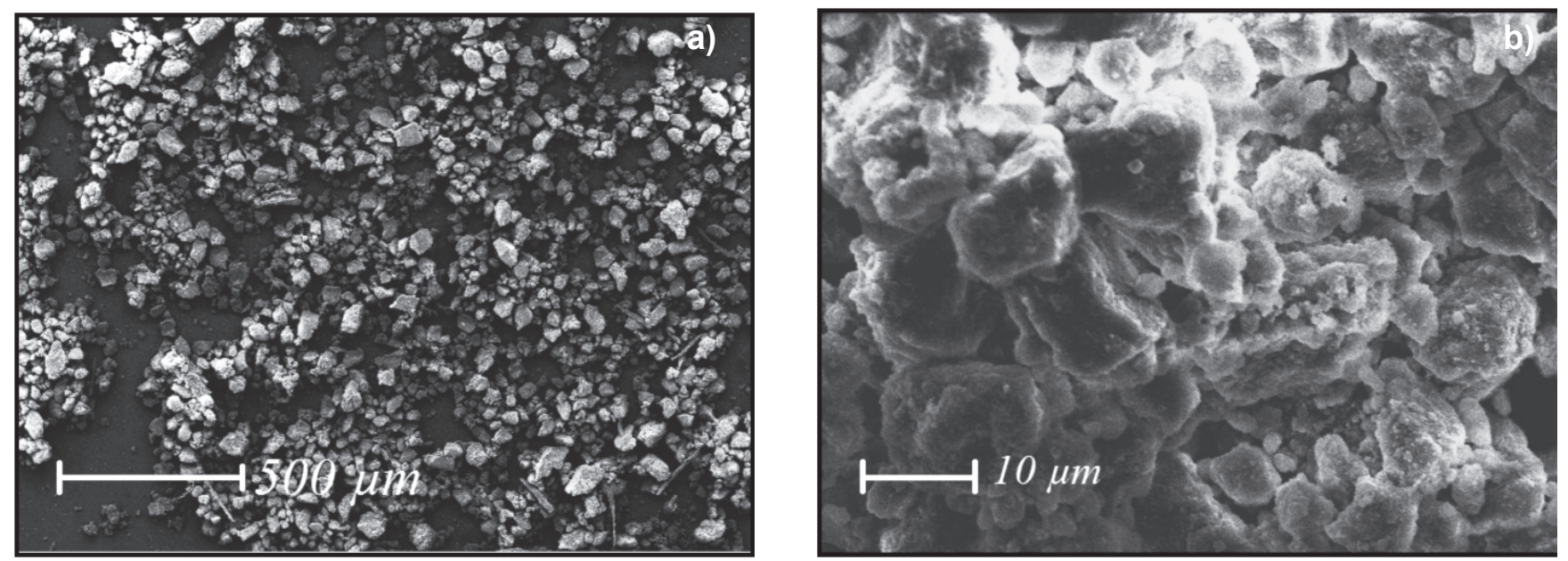

Figura 5: Micrografias obtidas por MEV. (a) Geral da amostra identificando matéria orgânica. (b) Partículas lamelares conglomeradas. [Figure 5: SEM micrographs. (a) identifying organic matter. (b) lamellar particles conglomerated.]
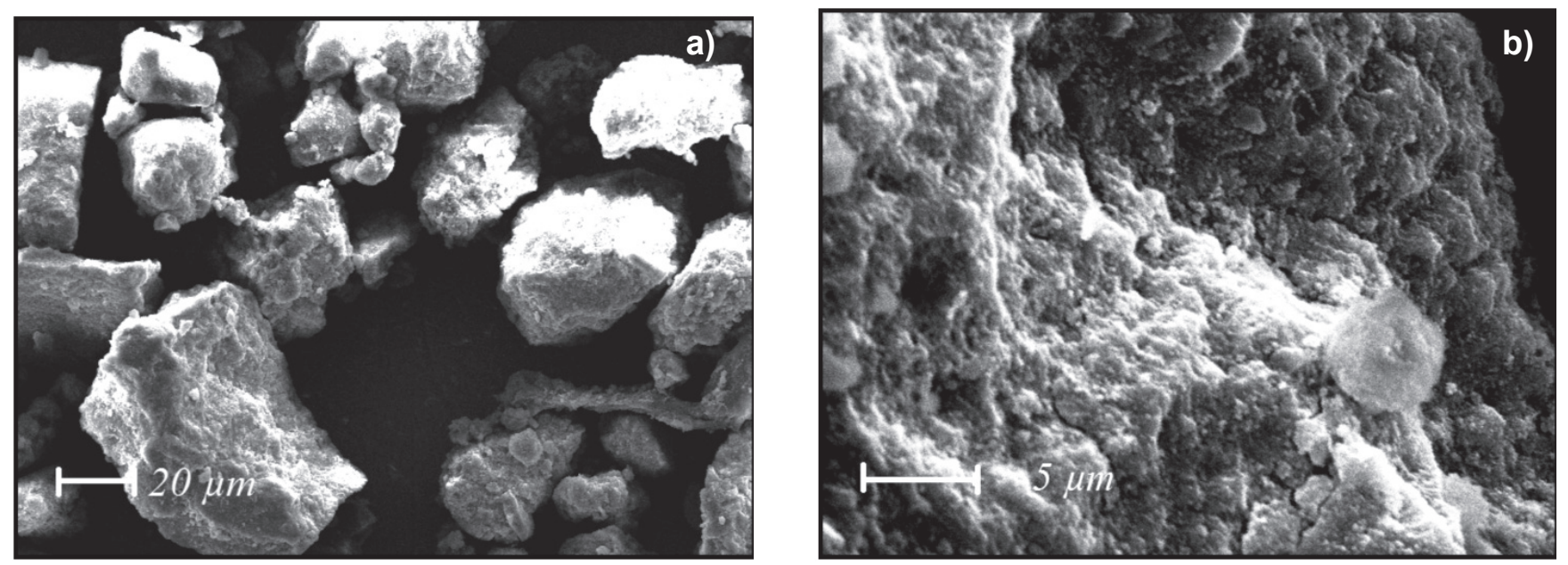

Figura 6: Micrografias obtidas por MEV. (a) Grânulos de lodo. (b) Superfície do grânulo.

[Figure 6: SEM micrographs. (a) Sludge granules. (b) Surface of the bead.]
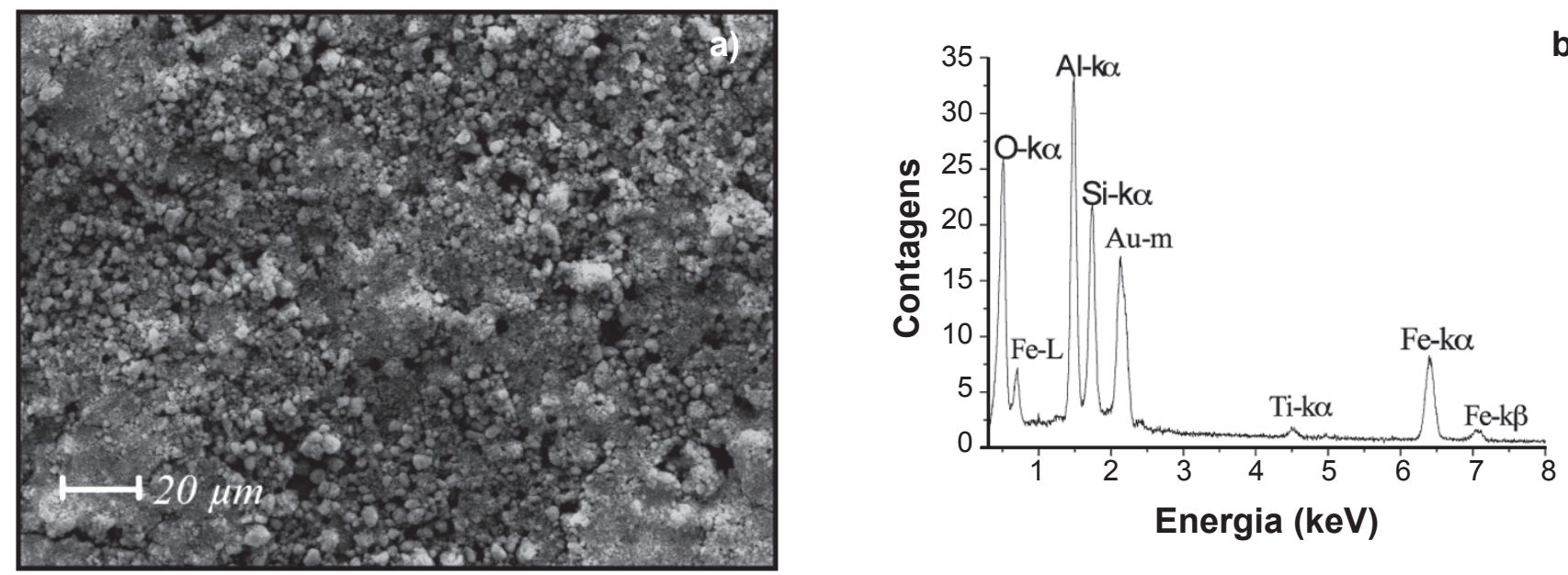

Figura 7: Micrografias obtidas por MEV. (a) Particulas aglomerados. (b) EDS geral da amostra. [Figure 7: SEM micrographs. (a) Particles clusters. EDS of the sample.]

sem dimensão, formato e perfil padronizado. A micrografia da Fig. 5a apresenta o aspecto geral da amostra do lodo de ETA, destacando a existência de matéria orgânica aderida às faces das partículas. Na Fig. 5b, observa-se conglomeração de partículas lamelares nas microrregiões de distintos tamanhos, sem dimensão e formato padronizado. A Fig. 
6a mostra que as partículas não possuem forma definida, somente estrutura de grânulos conglomerados. A Fig. 6b apresenta os detalhes da topografia desigual da superfície dos grânulos com formato de placas lamelares e com perfil irregular, cujas partículas aglomeradas aparentam ter forças atrativas fracas. Na Fig. 7a, pode-se observar a estrutura geral da amostra, cuja composição apresentada na Fig. 7a, é majoritária de $\mathrm{Al}_{2} \mathrm{O}_{3}, \mathrm{SiO}_{2}, \mathrm{FeO}$ e $\mathrm{TiO}_{2}$.

\section{CONCLUSÕES}

O lodo gerado na Estação de Tratamento de Água da unidade Tamanduá de Foz do Iguaçu, PR, não pode ser usado como componente majoritário em massas cerâmicas, devido suas características de desplastificante, grande porcentagem de grãos de tamanho grosseiro e elevada perda ao fogo. As argilas OP e AP apresentaram propriedades de alta plasticidade, caracterizadas como sendo argilas inorgânicas com granulometria favorável para produção de cerâmica vermelha, e baixa perda ao fogo. As argilas AA e OA foram caracterizadas como argilas formadas por siltes inorgânicos de alta compressibilidade, com percentual de areia de quartzo de mediana plasticidade. A composição química majoritária das matérias-primas são formadas por $\mathrm{Al}_{2} \mathrm{O}_{3}$, $\mathrm{SiO}_{2}, \mathrm{Fe}_{2} \mathrm{O}_{3}$, entretanto em percentuais diferenciados, com destaque para o lodo com $31,6 \%$ de $\mathrm{Al}_{2} \mathrm{O}_{3}$ o que deve-se a influência do coagulante utilizado no processo convencional de tratamento da água. O lodo por si só não pode ser usado como componente majoritário em massas cerâmicas, porém pode ser incorporado como aditivo em massas argilosas. A empresa de Saneamento do estado do Paraná está sendo sinalizada para o gerenciamento de seus resíduos, portanto, a alternativa de incorporação de lodo à massa cerâmica é fundamental para solucionar a disposição final deste resíduo. Logo, a etapa 2 deste trabalho apresentará os resultados tecnológicos da incorporação do lodo nas argilas para produção de cerâmica vermelha.

\section{AGRADECIMENTOS}

Os autores agradecem a SANEPAR/SANETEC pelo financiamento a pesquisa, ao PTI-Tecnologia, à Fundação Araucaria e ao CNPq pelas bolsas de estudo de mestrado e iniciação científica concedidas.

\section{REFERÊNCIAS}

[1] C. A. Richter, Tratamento de lodos de estações de tratamento de água, Ed. Edgard Blucher Ltda., S. Paulo (2001).

[2] A. C. L. S. M. Meneses, C. L. M. Gadelha, T. T. V. Machado, T. M. V. Almeida, W. R. S. Junior, Anais do $23^{\circ}$ Cong. Bras. Eng. Sanit. Amb. (2005) 1-8.

[3] S. N. Monteiro, J. Alexandre, J. L. R. Margem, R. Sanches, C. M. F. Vieira, Construction Building Mater. 22 (2008) 1281-1287.

[4] E. M. Ferranti, G. R. Gehling, Anais do $23^{\circ}$ Cong. Bras.
Eng. Sanit. Amb. (2005) 1-7.

[5] E. M. S. Oliveira, S. Q. Machado, J. N. F. Holanda, Cerâmica 50, 316 (2004) 324-330.

[6] V. M. T. Junior, V. Mymrine, R. A. C. Ribeiro, H. A. Ponte, Anais $17^{\circ}$ CBECIMat - Congresso Brasileiro de Engenharia e Ciências dos Materiais, Foz do Iguaçu, PR (2006) 1881-1891.

[7] C. Hoppen, K. F. Portela, A. Joukoski, O. Baron, R. Franck, A. Sales, C. V. Andreoli, V. A. Paulon, Cerâmica 51, 318 (2005) 85-95.

[8] M. M. T. Moreno, D. Bartolomeu, R. H. C. Lima, Cerâmica 55, 335 (2009) 286-295.

[9] L. C. C. Paixão, D. C. R. Espinosa, J. A. S. Tenório, Cong. Bras. Ci. Tecnol. Resíduos e Desenvolvimento Sustentável, Florianópolis, SC (2004) 1866-1873.

[10] S. R. Teixeira, S. A. de Souza, N. R. Souza, P. Aléssio, G. T. A. Santos, Cerâmica 52, 323 (2006) 215-220.

[11] H. Mekki, A. Michael, B. Mourad, A. Emna. J. Hazardous Mater. 158 (2008) 308-315.

[12] P. R. S. Cardoso, Informações técnicas sobre o policloreto de alumínio, Resposta Técnica produzida pelo Serviço Brasileiro de Respostas Técnicas / SBRT06 de junho 2006. Disponível em < http://www.sbrt.ibict.br>, acesso em 02/09/2009.

[13] MINEROPAR, Minerais do Paraná S/A., Gerência de Fomento e Economia Mineral, Levantamento das Potencialidades Minerais dos Municípios: Foz do Iguaçu, Curitiba, PR (1990) 83p.

[14] DNER - Departamento Nacional de Estradas e Rodagens, Método de Ensaio n. 041, designada Solos preparação de amostras para ensaios de caracterização (1994).

[15] DNER, Departamento Nacional de Estradas e Rodagens, Método de Ensaio n. 213, designada Solos - determinação do teor de umidade (1994).

[16] EMBRAPA, Empresa Brasileira de Pesquisa Agropecuária, Método de análise solos, Rio de Janeiro (1997).

[17] ABNT, Associação Brasileira de Normas Técnicas NBR 6459, Solo-determinação do Limite de Liquidez: Rio de Janeiro, ABNT (1984) 6p.

[18] ABNT, Associação Brasileira de Normas Técnicas NBR 7180, Solo-determinação do Limite de Plasticidade Método de Ensaio, Rio de Janeiro, ABNT (1984b) 3p.

[19] A. M. Maliska. Microscopia eletrônica de varredura e microanálise (2003). Disponível em $<\mathrm{http}$ ://www.materiais. ufsc.br/lcm/web-MEV/MEV_Apostila.pdf $>$, acesso em 10/2008).

[20] P. S. Santos, Ciência e Tecnologia de Argilas. 2a Ed., Vol. 1, Edgar Blucher, S. Paulo, SP (1989).

[21] A. Barba, V. Beltán, C. Feliu, J. Gárcia, F. Ginés, E. Saches, Materias primas para la fabricacion de soportes de baldosas cerámicas, Instituto de Tecnologia CerámicaAICE, Castellón (1997).

[22] R. L. Gomes, J. E. Rodrigues, Eng. Geology 91 (2007) 265-278.

(Rec. 25/04/2010, Rev.06/07/2010, Ac. 20/08/2010) 